This is the peer reviewed version of the following article: Isr. J. Chem.

2013, 53, 901, which has been published in final form at http://

ReVieW may be used for non-commercial purposes in accordance With Wiley-VCH Terms

and Conditions for self-archiving

DOI: 10.1002/ijch.200((full DOI will be filled in by the editorial staff))

\title{
Gold-Catalyzed Alkynylation: Acetylene-Transfer instead of Functionalization
}

\author{
Jonathan P. Brand, Yifan Li and Jérôme Waser*[a]
}

Abstract: In the last fifteen years, gold has raised from the status of an inert noble metal to one of the most often used catalyst in synthetic chemistry. The functionalization of the triple bond of alkynes has been especially successful in this respect. In contrast, gold-catalyzed alkynylation methods have begun to emerge only in 2007. Since then, three different approaches have been successful. Gold nanoparticles allowed catalytic cycles based on oxidative arylation on aryl halides to give a "palladium-free Sonogashira reaction". The use of benziodoxol(on)e hypervalent iodine compounds as oxidative alkynylation reagents had then allowed the $\mathrm{C}-\mathrm{H}$ functionalization of electron-rich heterocycles under mild conditions and with a very broad functional group tolerance. Finally, the use of iodobenzene acetate or Selectfluor as external oxidant led to the first alkynylation methods based on direct $\mathrm{C}-\mathrm{H} / \mathrm{C}-\mathrm{H}$ coupling. In only six years, gold-catalyzed alkynylation methods have raised from nonexistent to useful synthetic protocols for the synthesis of structurally diverse alkynes. When considering that acetylenes are among the most important building blocks for applications in synthetic chemistry, chemical biology and materials science, a tremendous potential for further development of gold-catalyzed alkynylation can be expected for the future.

Keywords: Gold • Alkynes • Catalysis • C-H Functionalization • Hypervalent lodine

\section{Introduction and Pioneering Works}

Gold as the noble metal per excellence has long been considered too inert to display interesting catalytic activity. It is only in the last fifteen years that organic chemists became truly aware of the tremendous potential of gold catalysis for the transformation of organic molecules. ${ }^{[1]}$ First success was achieved when gold was used for the activation of non-polarized $\pi$ systems, in particular acetylenes. ${ }^{[2]}$ Nowadays, cyclizations and cycloisomerizations based on gold catalysis constitute some of the best methods for a rapid access to molecules with complex carbon skeleton. ${ }^{[3]}$ Recently, the versatility of gold catalysis has been further increased by the discovery of new modes of activation based

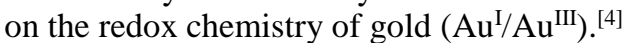

Right from the beginning, the activation of the triple bond of alkynes has been one of the most successful transformations in gold catalysis. This raises the interesting question whether gold catalysis could be used for the transfer instead of the functionalization of acetylenes. This question is not only interesting from the point of view of reactivity, but it is also of high practical relevance, when considering that alkynes are one of the most useful functional groups in synthetic chemistry, chemical biology and materials science. ${ }^{[5]}$

In fact, a first example of gold mediated alkynylation was already reported by Fuchita and co-workers in 2001 (Scheme 1). ${ }^{[6]}$ Stable aryl-Au ${ }^{\text {III }}$ lutidine complexes $\mathbf{1}$ were easily accessed from $\left[\mathrm{AuCl}_{3}\right]_{2}$ (1). The reaction of the obtained complexes with phenyl acetylene then gave the $\mathrm{C}-\mathrm{H}$ alkynylation products $\mathbf{2 a}$ and $\mathbf{2} \mathbf{b}$ in high yields
(2). Although not discussed extensively in the original publication, this second step probably involved formation of a $\mathrm{Au}^{\mathrm{III}}$-acetylene complex followed by reductive elimination to give a non-identified $\mathrm{Au}^{\mathrm{I}}$ product.

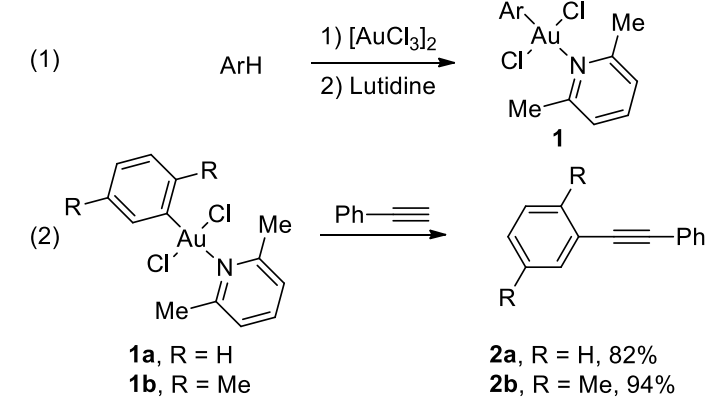

Scheme 1. Gold-mediated stoichiometric C-H alkynylation.

In principle, if an adequate oxidant could be found, the two steps could be combined in a catalytic cycle with a gold catalyst. In fact, this approach was attempted by Reetz and Sommer in 2003 (Scheme 2). ${ }^{[7]}$

\footnotetext{
[a] Yifan Li, Dr. Jonathan P. Brand and Prof. Dr. J. Waser Laboratory of Catalysis and Organic Synthesis Ecole Polytechnique Fédérale de Lausanne EPFL SB ISIC LCSO, BCH 4306, 1015 Lausanne $(\mathrm{CH})$ Fax: (+)41216939700

E-mail: jerome.waser@epfl.ch Homepage: http://lcso.epfl.ch/
} 
Despite extensive investigation on diverse oxidants, the desired alkynylation product was not observed. Instead, hydroarylation occurred, and the authors further optimized the latter transformation by using cationic gold complexes. This outcome was rationalized by the fact that $\pi$ activation of the alkyne was favoured over formation of the gold-aryl-alkynyl complex.

Whereas the work of Fuchita and co-workers has demonstrated that alkynylation based on a gold-redox mechanism is possible, the difficulties encountered by Reetz and Sommer highlighted the fundamental challenge of reactivity associated with this transformation. One approach would be to simplify the problem by using an oxidative (electrophilic) arylation or alkynylation reagent (A and $\mathbf{B}$ in Scheme 3 ). An external oxidant is not required in this case, making the system more general. Both approaches were indeed successful and will be discussed in section 2.1 and 2.2 of this review. Finally, if the right oxidant could be found for the direct $\mathrm{C}-\mathrm{H} / \mathrm{C}-\mathrm{H}$ coupling, more efficient methods would become available ( $\mathbf{C}$ in Scheme 3 ). Indeed, the very first examples of such transformations have appeared recently and will be discussed in section 2.3 of this review.

$$
\mathrm{Ar}-\mathrm{H} \underset{\mathrm{Au}(\mathrm{III}) \text { cat. }}{\stackrel{\equiv}{\equiv} \mathrm{Ph}}=\underbrace{\mathrm{Ph}}_{\mathrm{Ar}} \mathrm{Ar}=\mathrm{Ph}
$$

Scheme 2. Attempts towards gold-catalyzed alkynylation.

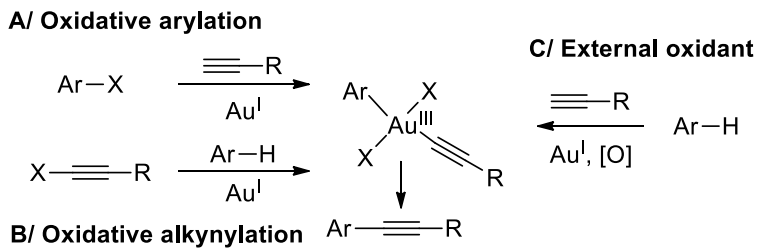

Scheme 3. Possible approaches for accessing gold-alkynyl-aryl intermediates.

Jonathan Brand was born in
Strasbourg, France in 1984. He studied
chemistry at the Ecole Nationale
Supérieure de Chimie de Montpellier
(France) and graduated in 2008 with
internships Actelion,
GlaxoSmithKline and Hoffmann-La
Roche. He was awarded the
"GlaxoSmithKline Certificate for
Outstanding Academic Achievement in
Organic Chemistry 2007-2008
Academic Year". In 2008 , he started his
PhD at EPF working on the direct
alkynylation of heterocycles using hypervalent iodine reagents
under the direction of Prof. Jérôme Waser. After obtaining his PhD
in 2012 with a special distinction of the EPFL research
commission, he accepted a position as a development chemist at
Givaudan.

Jonathan Brand was born in Strasbourg, France in 1984. He studied chemistry at the Ecole Nationale Supérieure de Chimie de Montpellier (France) and graduated in 2008 with PhD at EPFL working on the direct Givaudan.
Yifan Li was born in Jinan, China in 1986. He obtained his bachelor diploma in material chemistry in 2008 at Jinan University and then moved to the University of Lyon 1 where he obtained is master degree in organic chemistry in 2011. Since 2008, he is carrying out his $\mathrm{PhD}$ at Ecole Polytechnique Fédérale de Lausanne (Switzerland) working on the development of new catalytic methods using hypervalent iodine reagents under the direction of Prof. Jérôme Waser.

Jérôme Waser was born in Sierre, Valais, Switzerland in 1977. He studied chemistry at ETH Zurich and obtained his Diploma in 2001. In 2002, he started his PhD studies at ETH Zurich with Prof. Erick M. Carreira. In 2006, he joined Prof. Barry M. Trost at Stanford University. Since October 2007, he is working as tenure-track assistant professor at EPF Lausanne, focusing on the development and application of catalytic methods for the synthesis of bioactive compounds. His independent research has been recognized by the

Thieme Journal Award 2009, the A.F. Schläfli award 2011 of the Swiss Academy of Sciences and the Silver Medal of the European Young Chemist Award 2012

\section{Gold-Catalyzed Alkynylation}

\subsection{Catalytic Cycles Involving Oxidative Arylation}

The oxidative addition of an aryl electrophile onto $\mathrm{Pd}^{0}$ is one of the key steps of the classical Sonogashira reaction, arguably one of the most often used methods for the synthesis of alkynes. $\mathrm{Pd}^{0}$ and $\mathrm{Au}^{\mathrm{I}}$ both have the same $\mathrm{d}^{10}$ electronic configuration, which raises the question if a similar oxidative addition on $\mathrm{Au}^{\mathrm{I}}$ will be also possible. However, the $\mathrm{AuIII} / \mathrm{Au}^{\mathrm{I}}$ standard reduction potential is higher than the one of $\mathrm{Pd}^{\mathrm{II}} / \mathrm{Pd}^{0} \quad(1.41$ and 0.99 respectively), ${ }^{[8]}$ indicating that oxidative addition may be thermodynamically more difficult. Furthermore, $\mathrm{Cu}^{\mathrm{I}}$ plays an important role as co-catalyst in the Sonogashira reaction, via the formation of a copper-acetylide complex and transmetallation to $\mathrm{Pd}^{\mathrm{II}}$. A similar behaviour could also be expected from $\mathrm{Au}^{\mathrm{I}}$, allowing catalytic alkynylation using a single metal. ${ }^{[9]}$ In fact, such a reaction was first reported by Corma and co-workers for iodoarenes in 2007 (Scheme 4). ${ }^{[10]}$ A heterogenous gold/cerium oxide catalyst was first used, but simple $\mathrm{Au}^{\mathrm{I}}$ complexes, such as $\mathrm{AuCl}\left(\mathrm{PPh}_{3}\right)$ also displayed catalytic activity, leading to the speculation that $\mathrm{Au}^{\mathrm{I}}$ could be the active form of gold in the heterogenous catalyst. In 2008, Wang and co-workers reported a more efficient system using a $\mathrm{Au}^{\mathrm{I}}$ dppf catalyst. ${ }^{[11]}$

If we assume that $\mathrm{Au}^{\mathrm{I}}$ is really the active catalyst in these reactions, two different mechanisms could be considered (Scheme 5). In the first pathway, $\mathrm{Au}^{\mathrm{I}}$ would first undergo oxidative addition on iodobenzene (3) to give $\mathrm{Au}^{\mathrm{III}}$ aryl complex II. Base-mediated ligand exchange with phenyl acetylene (4) would then lead to aryl-alkynyl intermediate III. Reductive elimination would finally give $\mathrm{Au}^{\mathrm{I}}$ complex $\mathrm{I}$ and alkynylation 
product 5. The pioneering work of Fuchita supported the two steps from II to III and III to I. On the other hand, the base-mediated formation of $\mathrm{Au}^{\mathrm{I}}$ alkyne complex IV is known to be easy, as demonstrated by the fact that $\mathrm{Au}^{\mathrm{I}}$ catalysts have been used as an efficient substitute of $\mathrm{Cu}^{\mathrm{I}}$ in the $\mathrm{Pd}^{0}$-catalyzed Sonogashira reaction. ${ }^{[12]}$ Oxidative addition of complex IV onto iodobenzene (3) would then lead to the same intermediate III.

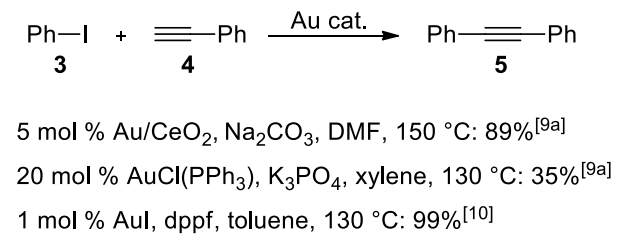

Scheme 4. Gold-catalyzed alkynylation of iodobenzene.

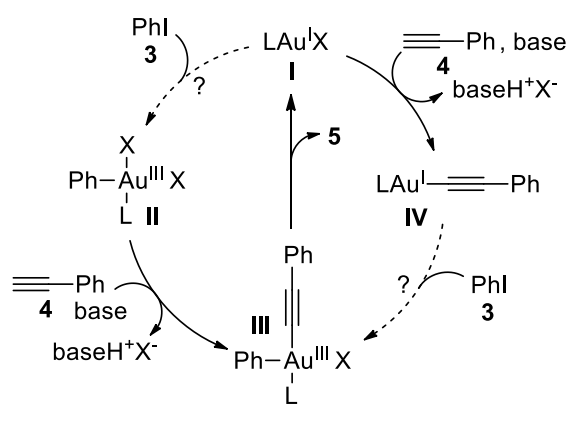

Scheme 5. Possible mechanisms for the gold-catalyzed alkynylation of aryl halides.

In 2010, Echavarren and co-workers studied the two possible catalytic cycles more in detail. ${ }^{[13]}$ They first could confirm the result obtained by Fuchita for the easy reductive elimination from $\mathrm{Au}^{\mathrm{III}}$ alkynyl-aryl complexes. However, when the two possible alternatives for oxidative addition of $\mathrm{Au}^{\mathrm{I}}$ onto iodobenzene (3) were examined stoichiometrically, using either $\mathrm{Au}^{\mathrm{I}}$ alkynyl phosphine complex $\mathbf{6}$ or $\mathrm{Au}^{\mathrm{I}}$ chloride phosphine complex 7, no reaction was observed (Scheme 6, (1) and (2)). This result makes a catalytic cycle based on a $\mathrm{Au}^{\mathrm{I}}$ catalyst highly unlikely. The observed catalytic activity could then be either coming from palladium impurities or from another oxidation state of gold. Indeed, in-depth investigations by Lambert and co-workers ${ }^{[14]}$ and Corma and co-workers ${ }^{[15]}$ demonstrated that the active catalyst for oxidative addition on iodobenzene was probably $\mathrm{Au}^{0}$ nanoparticles. Such nanoparticles can also be formed in situ when starting from homogenous $\mathrm{Au}^{\mathrm{I}}$ catalysts. ${ }^{[14 \mathrm{c}]}$ Interestingly, a synergic effect between $\mathrm{Au}^{0}$ for oxidative addition and $\mathrm{Au}^{\mathrm{I}}$ for activation of the terminal acetylene can also be proposed in the case of heterogenous catalysts. ${ }^{[15 \mathrm{~b}]}$

A last interesting contribution to the elucidation of the reaction mechanism was made in 2012 by O'Hair and co-workers, who studied again the stoichiometric step of the catalytic cycle using mass spectrometry and calculations (Scheme 7). ${ }^{[16]}$ Using a bis-ligated cationic gold complex 8, they did not observe any product resulting from oxidative addition by mass spectrometry (1). Calculations confirmed that this reaction was endothermic and had a high activation energy. In contrast, products resulting from oxidative addition were observed both with a mono-ligated $\mathrm{Au}^{\mathrm{I}}$ complex 10 (2) and a trinuclear $\mathrm{Au}$ cluster 12 (3). Calculations showed that both reactions were now exothermic and the activation barrier was lower, but much strongly so in the case of the cluster. Although care has to be taken to translate these results to the conditions of catalytic reactions, they further confirmed the importance of $\mathrm{Au}^{0}$ for oxidative addition on iodobenzene (3).
(1)
Scheme 6. Investigation on stoichiometric reactions for the oxidative addition of $\mathrm{Au}^{\prime}$ complexes on iodobenzene (3).

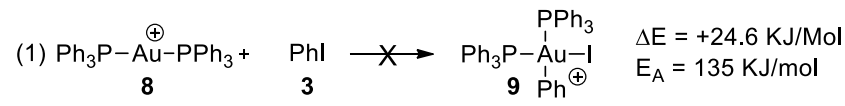

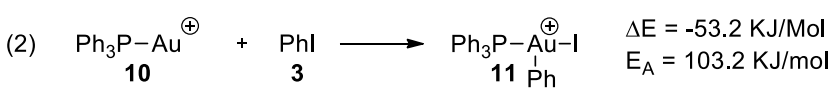

$$
\begin{aligned}
& \text { (3) } \quad \mathrm{LAu}_{3}^{\oplus}+\mathrm{Phl} \longrightarrow \begin{array}{cl}
\mathrm{LAM}_{3} \mathrm{Phl} & \Delta \mathrm{E}=-182.9 \mathrm{KJ} / \mathrm{Mo} \\
\mathrm{E}_{\mathrm{A}}=80.3 \mathrm{KJ} / \mathrm{mol}
\end{array} \\
& \mathrm{L}=\mathrm{Ph}_{2} \mathrm{P} \sim \mathrm{PPh}_{2}^{13} \sim^{13}
\end{aligned}
$$

Scheme 7. Combined mass spectrometry and computational studies for the oxidative addition of $\mathrm{Au}$ complexes on iodobenzene (3).

In summary, these fascinating studies on the "goldcatalyzed Sonogashira" reaction have significantly increased our understanding of the mechanism of oxidative addition of gold on aryl halides. The current conscientious is that although homogenous $\mathrm{Au}^{\mathrm{I}}$ catalysts cannot undergo oxidative addition on aryl halides, this reaction is possible for $\mathrm{Au}^{0}$ nanoparticles, opening the way to the catalytic alkynylation of arene halides using heterogenous gold catalysts. Although it may be possible to achieve oxidative addition on $\mathrm{Au}^{\mathrm{I}}$ complexes using stronger oxidant, such as aryl iodonium salts, such attempts have not yet been reported to the best of our knowledge.

\subsection{Catalytic Cycles Involving Oxidative Alkynylation}

The development of the gold-catalyzed alkynylation of aryl halides was fascinating from the mechanism point of view, but this transformation will always be in competition with the often very efficient palladium-catalyzed Sonogashira reaction. On the other hand, the use of an oxidative alkynylation method would lead to the direct $\mathrm{C}-\mathrm{H}$ functionalization of arenes via an "inverse Sonogashira" reaction. ${ }^{[17]}$ Such a transformation is highly attractive, as the synthesis of aryl halides would be avoided. Furthermore, they are only few methods based on palladium or copper catalysts to achieve such transformations. ${ }^{[18]}$ Nevertheless, 
the pioneering work of Reetz and co-workers had demonstrated that such a reaction would be difficult to achieve. ${ }^{[7]}$

As in situ oxidation has not been successful to promote alkynylation in the hand of Reetz and coworkers, our group decided to use well-defined electrophilic alkynylation reagents and indole as one of the most important electron-rich heterocycles as substrate. As no reaction was observed when using alkynyl halides, more reactive alkynyliodonium salts ${ }^{[19]}$ were examined next. Again, this reaction was not successful: full conversion of the alkynylation reagent was observed, but only diynes were obtained as product. It is only when a more stable cyclic hypervalent iodine reagent, 1-[(triisopropylsilyl)ethynyl]-1,2-benziodoxol3(1H)-one (TIPS-EBX, 15) was used together with $\mathrm{AuCl}$ as catalyst that $\mathrm{C} 3$-alkynylation of indole 14a was obtained with complete selectivity in $84 \%$ yield (Scheme 8). ${ }^{[20]}$ Later studies demonstrated that both the benziodoxolone core and a bulky silyl group on the alkyne were required for high yields. ${ }^{[21]}$ Although the requirement for a bulky silyl group constituted a limitation of the method, the obtained silylated alkynes could be easily deprotected to give access to the most synthetically versatile terminal acetylenes.

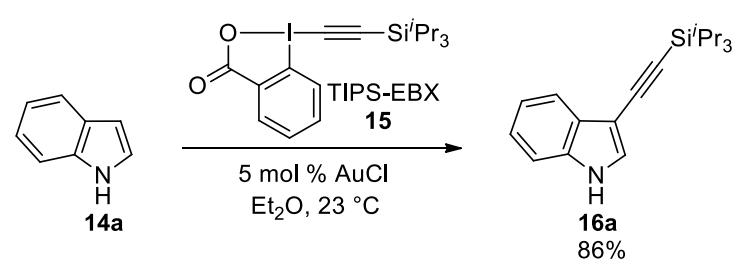

Scheme 8. First example of $\mathrm{Au}(\mathrm{I})$-catalyzed alkynylation using TIPS-EBX (15).

The developed protocol is very robust and easy to use, as the reaction is not moisture or oxygen sensitive and can be run under open flask conditions. Many solvents are tolerated in the reaction, which proved to be important to extend the scope of the reaction (Scheme 9). With indoles, the reaction worked best in diethyl ether, probably because the reagent is only slightly soluble in this solvent, preventing a too fast reaction. For all substrates, high selectivity for the most electron-rich position was observed, and the reaction kinetics was correlated to the nucleophilicity of the heterocycle, as given for example by the Mayr's factor. ${ }^{[22]}$

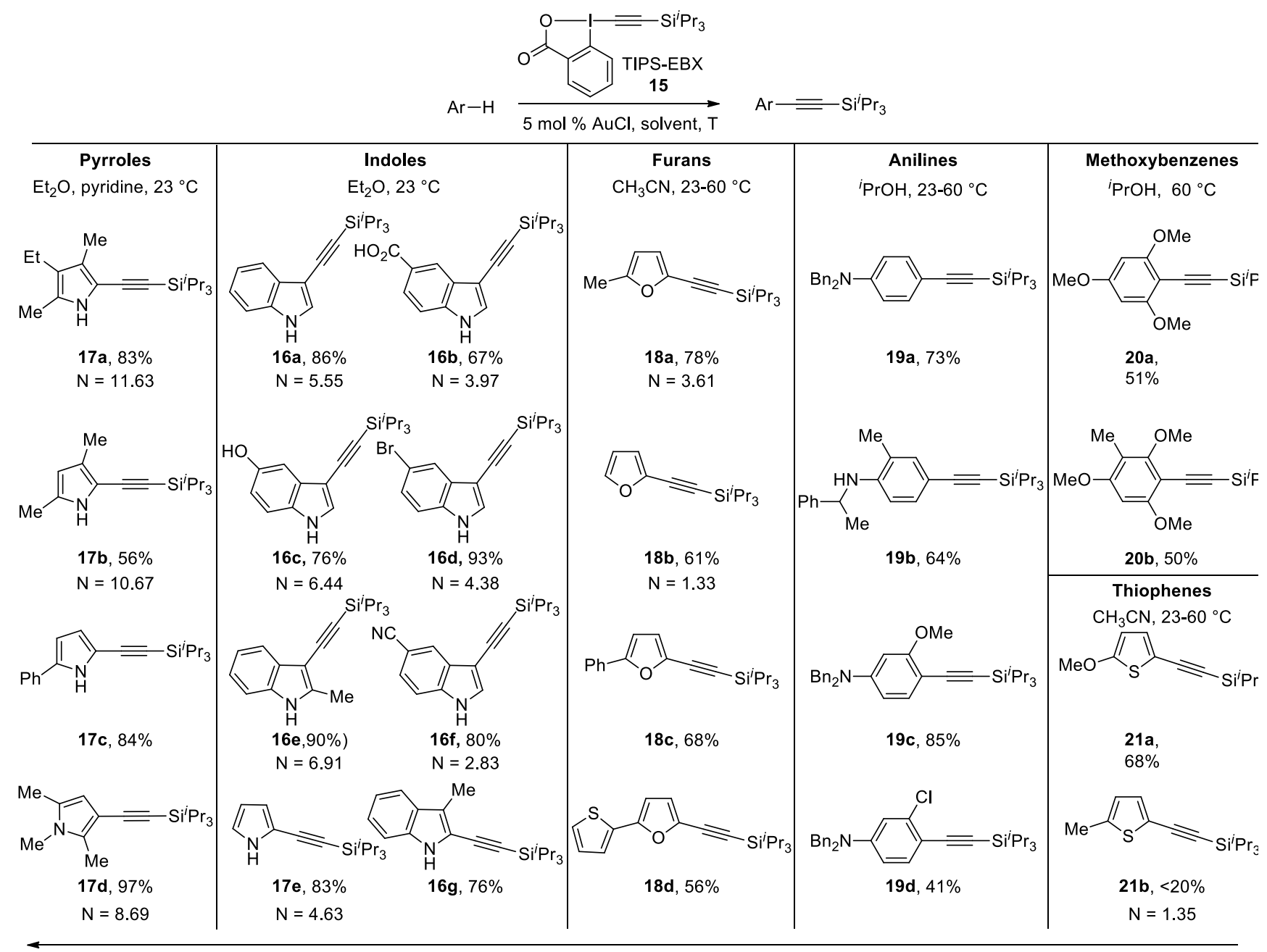

NUCLEOPHILICITY

Scheme 9. Selected examples of $\mathrm{C}-\mathrm{H}$ alkynylation. If available, the nucleophilicity factor $\mathrm{N}$ according to the Mayr's scale is given. 
An important advantage of the gold-catalyzed alkynylation is the broad functional group tolerance. On indole for example, carboxylic acids (16b), phenols (16c), halogens (16d) and cyano (16f) groups were tolerated. The reaction consequently worked in the presence of electron-rich and electron-poor substituents, heteroatoms and acidic hydrogens. As halogens remained untouched, the method is also orthogonal to classical cross-coupling chemistry. C2 or C3substituted indoles could also be used (products 16e and 16g). In the latter case, $\mathrm{C} 2$ alkynylation was obtained.

For more nucleophilic pyrroles, decomposition of the starting material led to lower yields under the same conditions. ${ }^{[20 \mathrm{a}]}$ Fortunately, the addition of pyridine as additive prevented degradation and the alkynylation products could be isolated in high yields, even in the case of very electron-rich tetrasubstituted pyrrole 17a. ${ }^{[21]}$ In this case, pyridine is probably acting as a ligand to diminish the Lewis acidity of gold, as no effect was observed when 2,6-di-tert-butyl pyridine was used as additive.

For less nucleophilic substrates, such as furans ${ }^{[23]}$ or anilines, ${ }^{[24]}$ complete conversion could not be achieved in diethyl ether as solvent. Fortunately, the tolerance of the catalyst and hypervalent iodine reagent to polar solvents allowed the use of acetonitrile or isopropanol, which led to a faster reaction. For the slowest reacting substrates, mild heating up to $60{ }^{\circ} \mathrm{C}$ was required to obtain good yields. Again, both classes of substrates reacted at the position with the highest electron density: $\mathrm{C} 2$ of furans and $\mathrm{C} 4$ of anilines. In the case of anilines, a moderately bulky group (benzyl) on the nitrogen was important to prevent competitive ortho alkynylation. Finally, when using even less nucleophilic substrates such as methoxybenzenes or thiophenes, complete conversion could never be achieved, even under forcing conditions. Alkynylated thiophene 21a constituted an exception, due to the electron-rich methoxy substituent. Consequently, the reaction could be applied successfully to a broad range of substrates with a nucleophilicity of 2 to 12 on the Mayr's scale.

Alkynylation of less nucleophilic substrates like thiophenes was not possible using the developed method. This was an important shortcoming, as alkynyl thiophenes are particularly valuable in the field of organic materials. ${ }^{[25]}$ It is known that the reactivity of hypervalent iodine reagents can be further increased by Lewis or Brønsted acid activation, ${ }^{[26]}$ and we wondered if this approach would be compatible with gold catalysis. Indeed, this was the case, and excellent yields could be obtained for a broad range of thiophenes using trifluoroacetic acid (TFA) as co-activator (Scheme 10). Again, the reaction was tolerant to functional groups containing heteroatoms (21d), aromatic rings (21e) or halogens (21f). It gave access to important alkynylated building blocks for materials science, such as bithiophene $21 \mathrm{f}$ or bis-alkynylated EDOT 21g. Finally, the reaction could also be applied to benzothiophenes, although the presence of a substituent in C3 position was required in this case to prevent the formation of regioisomers (product $\mathbf{2 1 h}$ ).
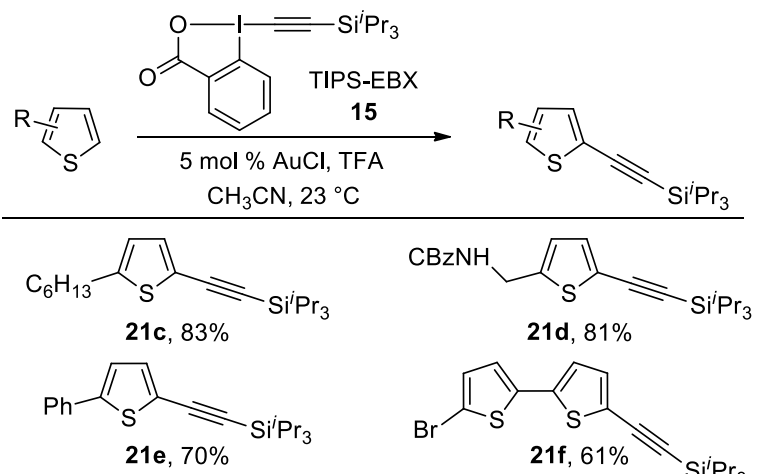
21d, $81 \%$
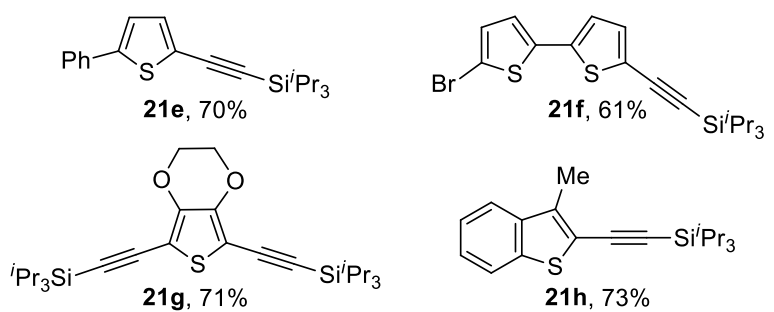

Scheme 10. Cooperative Brønsted acid-gold catalysis for the alkynylation of thiophenes.

Elucidation of the mechanism of the gold-catalyzed alkynylation with TIPS-EBX (15) is challenging. In particular, any electron-donating ligand such as a phosphine or a carbene cannot be used, as the resulting gold complexes are not active for alkynylation. As a fast reaction was observed between $\mathrm{AuCl}$ and the reagent to give mostly the diyne product, the first step is probably activation of the reagent by the catalyst to give complex I (Scheme 11).

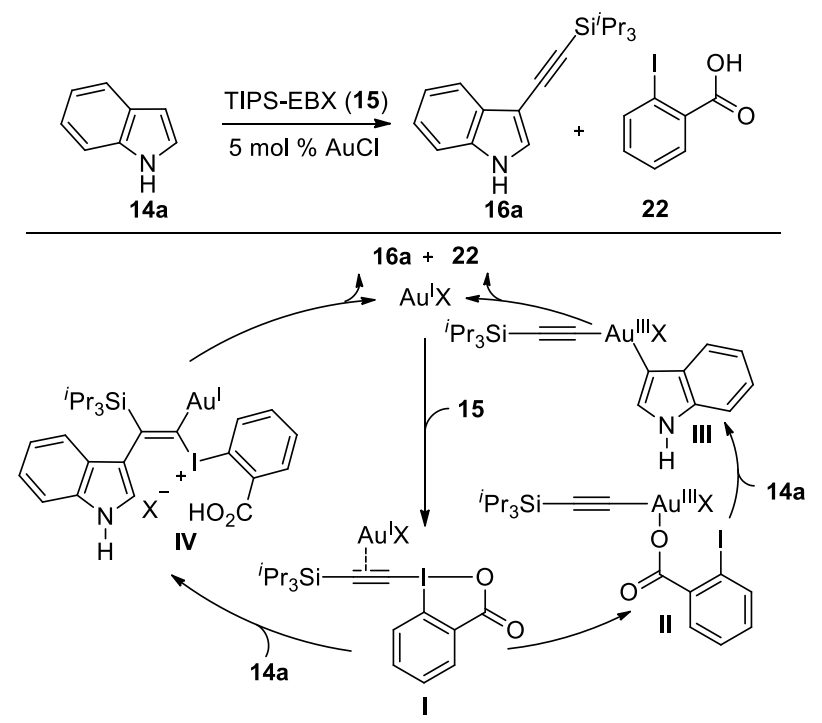

Scheme 11. Speculative mechanism for the alkynylation of indoles.

At this point, oxidative addition of $\mathrm{Au}^{\mathrm{I}}$ on the hypervalent iodine could be expected to give $\mathrm{Au}^{\text {III }}$ alkyne complex II. Auration of indole at the most electron-rich position followed by reductive elimination on III will then give the alkynylation product. Key to success would be the stronger oxidative power of hypervalent iodine reagents when compared with simple 
halides. In fact, no reaction was observed when iodoalkynes were used in the reaction. Nevertheless, direct Friedel-Crafts like addition of the indole onto the reagent to give $\mathbf{I V}$ without formation of a $\mathrm{Au}^{\mathrm{III}}$ alkynyl complex cannot be excluded at this stage. The very high leaving group ability of hypervalent iodine would explain in this case why $\alpha$-elimination followed by 1,2 indole shift would be preferred over hydroarylation. Addition to the triple bond with reverse regiochemistry followed by $\beta$-elimination could also be conceived, but it appeared less probable due to the very high polarisation induced by the hypervalent iodine atom. Finally, auration of indole as a first step or the involvement of gold nanoparticles would constitute further possible alternatives for the catalytic cycle.

The very high selectivity for the alkynylation of the most electron-rich position of heterocycles was useful for predicting the outcome of the reaction. On the other hand, it made the alkynylation of other positions very challenging. A solution would be to generate an organogold intermediate by a method not involving an electrophilic aromatic substitution mechanism. Especially interesting would be a domino-cyclizationalkynylation process making use of the well-established catalytic properties of gold complexes for the activation of alkynes or allenes in the synthesis of heterocycles. ${ }^{[2}$, 27] A proof of principle for this approach was first attempted in the case of the one-step synthesis of 3alkynyl indoles starting from ortho-alkynyl anilines. However, it was not possible to promote the domino process with a single gold catalyst. The use of a one-pot method for this sequence using a $\mathrm{Au}^{\mathrm{III}}$ and a $\mathrm{Au}^{\mathrm{I}}$ catalyst was finally successful (Scheme 12). ${ }^{[28]}$ The reaction worked well for aryl and alkyl substituents on the alkynes (products $\mathbf{2 4 a - e ) , ~ b u t ~ c o u l d ~ n o t ~ b e ~ u s e d ~ i n ~ t h e ~}$ case of terminal acetylenes (product $\mathbf{2 4 f}$ ). This work demonstrated the challenge represented by the development of a true domino cyclization-alkynylation process, as only a one-pot sequence of cyclization and alkynylation could be achieved.

Allene ketones would represent another interesting class of substrates for a domino-cyclization process (Scheme 13). In fact, gold catalysis has been highly successful for their cyclization. ${ }^{[2 \mathrm{~b}]}$ This reaction would also have the advantage to give access to $\mathrm{C} 3-$ alkynylated furans, which cannot be accessed via the $\mathrm{C}$ $\mathrm{H}$ functionalization methodology. However, when allene ketones were submitted to the conditions used for $\mathrm{C}-\mathrm{H}$ alkynylation, no reaction was observed, and the nonalkynylated furan was obtained when using the conditions reported for cyclization. A broader screening of reaction conditions, gold catalysts and hypervalent iodine reagents led finally to the unique combination of picolinic acid $\mathrm{Au}^{\mathrm{III}}$ complex 26, benziodoxole 27 and sodium carbonate as base. ${ }^{[23]}$ The presence of base was important to prevent premature protonation of the organogold intermediate, and fine-tuning of the electronic properties of the hypervalent iodine reagent was essential for the efficiency of the reaction. The domino process worked well for electron-neutral (product 28a) and electron-rich arenes (products 28b-d), as well as alkyl groups as substituents (products $\mathbf{2 8 e - g}$ ). A substituent at the $\alpha$ position to the carbonyl was also well tolerated, giving access to trisubstituted furan $\mathbf{2 8 h}$ with complete regioselectivity.
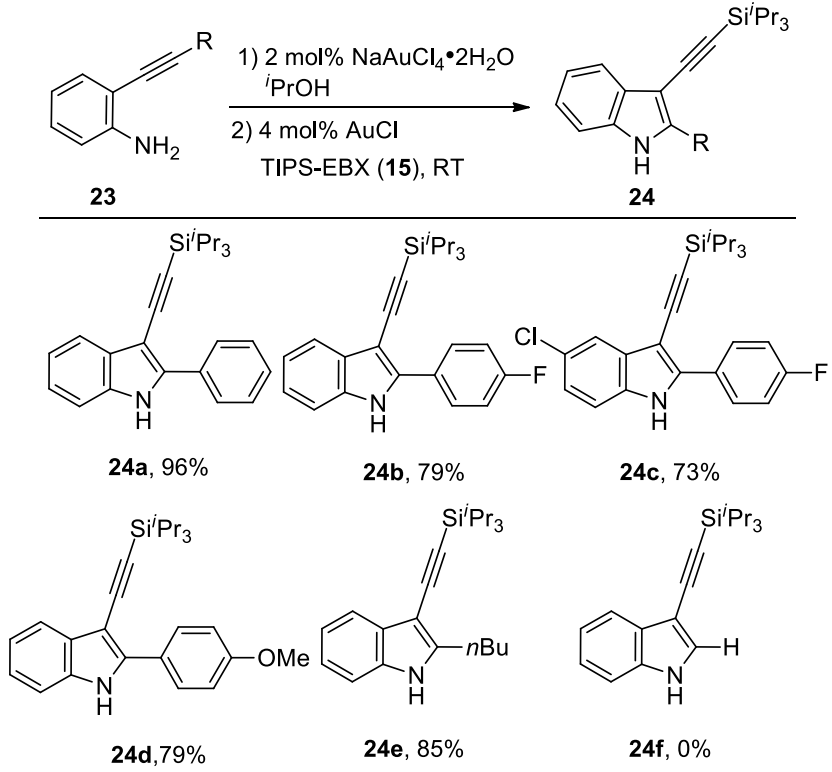

Scheme 12. One-pot synthesis of 3-alkynyl indoles starting from ortho-alkynyl anilines.

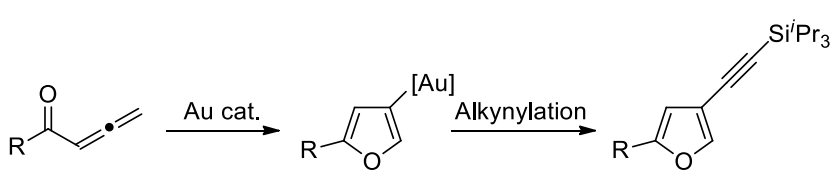

Scheme 13. Domino cyclization-alkynylation of allene ketones.

Control experiments showed that no $\mathrm{C}-\mathrm{H}$ alkynylation of furans was possible under the developed conditions. Furthermore, gold complex $\mathbf{2 6}$ did not react with benziodoxole 27. In contrast, a very fast cyclization reaction was observed when $\mathbf{2 6}$ was mixed with allene 25. These experiments supported the fact that the reaction is a true domino process. Finally, both the picolinic ligand and $\mathrm{a} \mathrm{Au}^{\mathrm{III}}$ catalyst were required, as no product was obtained with $\mathrm{Au}^{\mathrm{I}}$ or $\mathrm{Au}^{\mathrm{III}}$ complexes bearing other ligands.

In summary, the use of hypervalent benziodoxol(on)e reagents has made numerous new gold-catalyzed alkynylation reactions possible. Electron-rich indoles, pyrroles, anilines and furans could be alkynylated under mild conditions with simple $\mathrm{AuCl}$ as catalyst using commercially available TIPSEBX (15) as reagent. With a dual gold-Brønsted acid system, the alkynylation of thiophenes then become possible. Finally, the development of the first true domino cyclization-alkynylation process is very promising for the further use of acetylene transfer as a terminating step in gold-catalyzed cyclization reactions. 

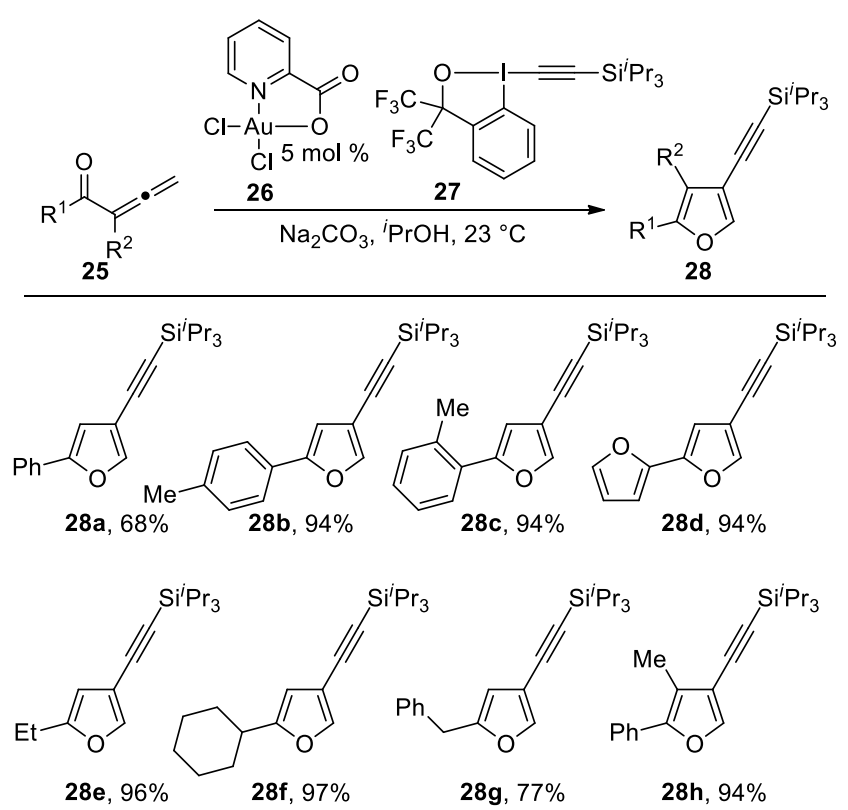

Scheme 14. Domino cyclization-alkynylation.

\subsection{Catalytic Cycles Involving Oxidative Coupling with an External Oxidant}

The development of gold-catalyzed alkynylation reactions based on direct $\mathrm{C}-\mathrm{H} / \mathrm{C}-\mathrm{H}$ coupling between arenes and terminal acetylenes is extremely challenging. First, the desired cross-coupling always competes with the homocoupling of either the arene or the alkyne. Second, the oxidation from $\mathrm{Au}^{\mathrm{I}}$ to $\mathrm{Au}^{\mathrm{III}}$ is difficult, as became apparent during the investigations of Reetz and Sommer. ${ }^{[7]}$ Finally, the competing hydroarylation reaction needs to be suppressed. An important breakthrough in the area was achieved by de Haro and Nevado in 2010 (Scheme 15). ${ }^{[29]}$ By using a simple $\mathrm{Au}^{\mathrm{I}}$ phosphine catalyst and iodobenzene diacetate as external oxidant, they were able to develop the first alkynylation of methoxy-substituted benzenes with electron-deficient alkynes. The reaction worked well for both ester and ketone substituted acetylenes and for benzene rings bearing at least two alkoxy substituents (products 29ad). Finally, the reaction could also be used for the alkynylation of other electron-rich aromatics, as for example indole $\mathbf{3 0}$ and chromene $\mathbf{3 1}$.

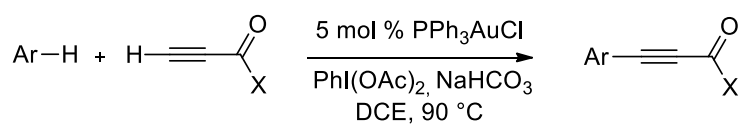

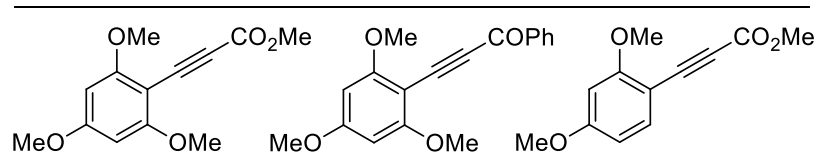

$29 a, 81 \% \quad 29 b, 72 \%$

29c, $52 \%$

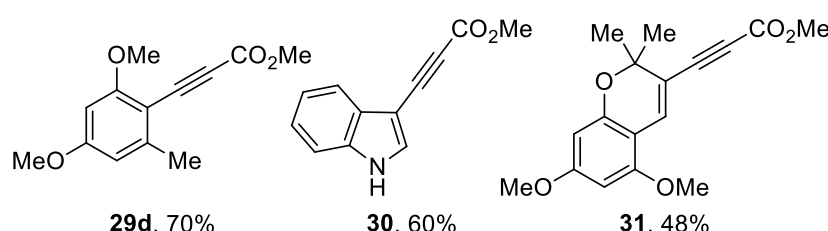

The first step in the catalytic cycle was proposed to be the formation of $\mathrm{a} \mathrm{Au}^{\mathrm{I}}$ acetylide complex II, which was in fact detected in the reaction mixture by NMR. At this stage, iodobenzene diacetate could oxidize intermediate II to $\mathrm{Au}^{\mathrm{III}}$ complex III. Electrophilic auration followed by reductive elimination would then give the alkynylation product. Alternatively, alkynylation of the iodine atom could first occur to give alkynyliodonium $\mathbf{V}$. $\pi$-activation of $\mathbf{V}$ by the goldcatalyst would lead to a Friedel-Crafts type addition of the arene. At this point, the regioselectivity of the addition on the triple bond is not so clear, due to the competing influence of the iodine and the ester. Formation of regioisomer VI would lead easily to the product via a $\beta$-elimination pathway, whereas for the other regioisomer a $\alpha$-elimination 1,2-shift sequence could be considered. Finally, it is also possible that intermediate $\mathbf{V}$ could oxidize gold to give $\mathrm{Au}^{\text {III }}$ complex III.

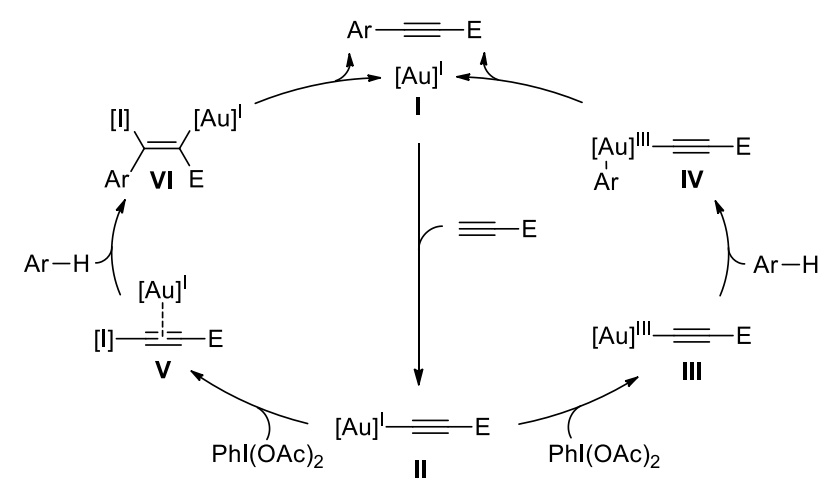

Scheme 16. Speculative mechanism for the $\mathrm{C}-\mathrm{H} / \mathrm{C}-\mathrm{H}$ coupling.

Also in 2010, Gouverneur and co-workers reported the first successful domino cyclization-oxidative alkynylation (Scheme 17) ${ }^{[30]}$ Starting from allene esters 32, this transformation gave directly access to alkynesubstituted butenolides 34. In this case, aromatic alkynes $\mathbf{3 3}$ were the best substrates, and a broad range of electron-rich and electron-poor substituents were tolerated on the alkyne. Key to success was the use of Selectfluor as an external strong oxidant to promote oxidation from $\mathrm{Au}^{\mathrm{I}}$ to $\mathrm{Au}^{\mathrm{III}}$. In several cases, the formation of the diyne resulting from oxidative dimerization of the alkyne was observed as a major side reaction. Two alternative mechanisms have been proposed for this transformation: $\mathrm{Au}^{\mathrm{I}}$-catalyzed cyclization, followed by oxidation by Selectfluor, formation of a $\mathrm{Au}^{\mathrm{III}}$ alkyne complex and reductive elimination or first oxidation of a $\mathrm{Au}^{\mathrm{I}}$ acetylide complex, followed by cyclization and reductive elimination. 


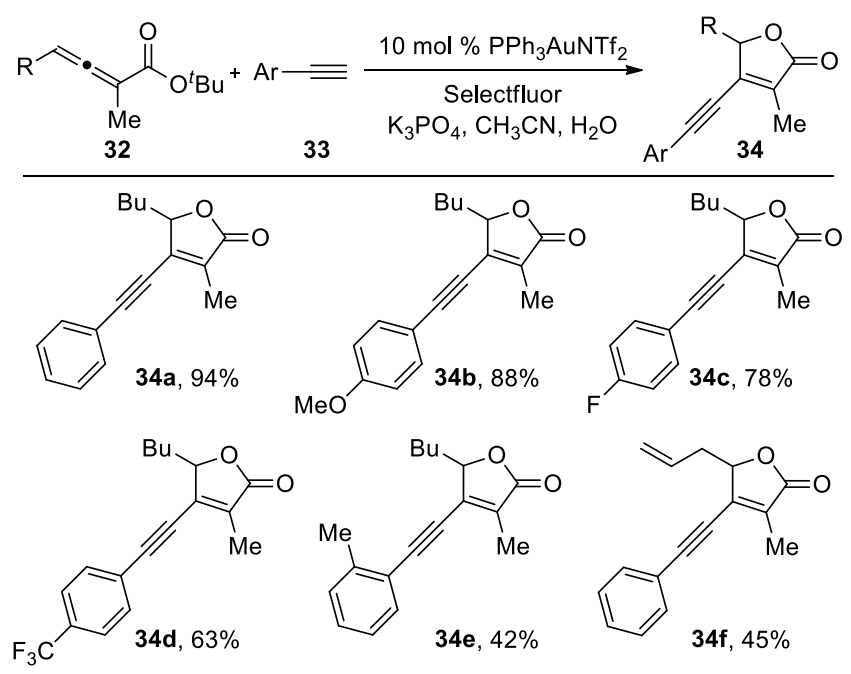

Scheme 17. Domino-cyclization alkynylation of allene esters

After these two seminal studies, Qian and Zhang developed in 2011 the gold-catalyzed oxidative coupling between aryl boronic acids $\mathbf{3 5}$ and terminal acetylenes 36 (Scheme 18). ${ }^{[31]}$ Although this transformation asked for the use of more expensive boronic acids as partners, it allowed for the first time the use of functionalized aliphatic alkynes. The alkynylation worked both for electron-poor and moderately electron-rich aryl boronic acids (products 37b and 37c). On the alkyne, it tolerated the presence of nitrogen (products 37a-c) and oxygen heteroatoms (products $\mathbf{3 7 d - e ) , ~ a s ~ w e l l ~ a s ~ a n ~ o l e f i n ~}$ (product 37f).

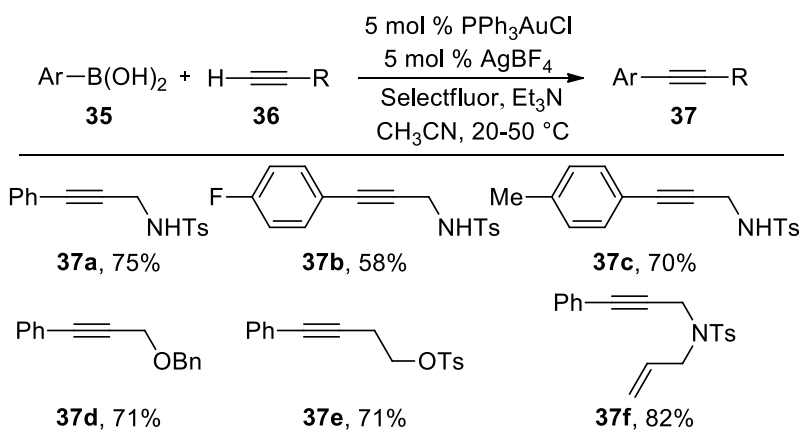

Scheme 18. Oxidative coupling of boronic acids with alkynes.

Finally, Corma and co-workers ${ }^{[32]}$ and $\mathrm{Fu}$ and coworkers $^{[33]}$ studied the gold-catalyzed dimerization of alkynes more in detail (Scheme 19, (1) and (2)). The Corma group used Selectfluor as oxidant, whereas Fu and co-workers used iodobenzene diacetate. In both cases, the reaction proceeded in good yields. Corma and co-workers proposed that a possible mechanism for the formation of the diyne is attack of a $\mathrm{Au}^{\mathrm{I}}$ acetylide complex onto a $\mathrm{Au}^{\mathrm{III}}$-alkynyl intermediate. The rate limiting step for the reaction was proposed to be the oxidation of $\mathrm{Au}^{\mathrm{I}}$ to $\mathrm{Au}^{\mathrm{III}}$ by Selectfluor.
(1)

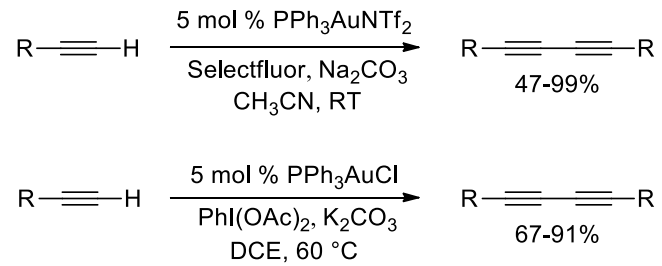

Scheme 19. Gold-catalyzed dimerization of alkynes.

To conclude this section, the first examples of goldcatalyzed alkynylation based on $\mathrm{C}-\mathrm{H} / \mathrm{C}-\mathrm{H}$ coupling have appeared very recently. Key to success was the identification of iodobenzene diacetate and Selectfluor as external oxidants. This breakthrough will allow in the future the development of more direct alkynylation methods proceeding without need for prefunctionalization of the substrates.

\section{Summary and Outlook}

Although the stoichiometric bases for gold-mediated alkynylation have been reported by Fuchita already in 2001 , it is only starting in 2007 that the first catalytic reactions could be developed. This required suppressing undesired side reactions, especially reaction on the triple bond of the alkyne and dimerization. Finally, success was achieved based on three different approaches. Gold nanoparticles allowed catalytic cycles based on oxidative arylation on aryl halides to give a "palladium-free Sonogashira reaction". From these studies, our understanding of the redox chemistry of gold has been greatly improved. The use of benziodoxol(on)e hypervalent iodine compounds as oxidative alkynylation reagents had then allowed the $\mathrm{C}$ $\mathrm{H}$ functionalization of electron-rich heterocycles under mild conditions and a very broad functional group tolerance. Preliminary results for the use of these reagents in gold-catalyzed domino processes are also very promising. Finally, the use of iodobenzene acetate or Selectfluor as external oxidant led to the first alkynylation methods based on direct $\mathrm{C}-\mathrm{H} / \mathrm{C}-\mathrm{H}$ coupling.

In only six years, gold-catalyzed alkynylation methods have raised from non-existent to useful synthetic protocols for the synthesis of structurally diverse alkynes. When considering that acetylenes are among the most important building blocks for applications in synthetic chemistry, chemical biology and materials science, a tremendous potential for further development of gold-catalyzed alkynylation can be expected in the future.

\section{Acknowledgements}

EPFL and F. Hoffmann-La Roche Ltd are acknowledged for financial support.

\section{References}

[1] a) A. S. K. Hashmi, G. J. Hutchings, Angew. Chem., Int. Ed. 2006, 45, 7896; b) A. S. K. Hashmi, Chem. Rev 2007, 107, 3180; c) D. J. Gorin, F. D. Toste, Nature 2007, 446, 
395; d) Z. G. Li, C. Brouwer, C. He, Chem. Rev 2008 108, 3239; e) T. de Haro, C. Nevado, Synthesis 2011, 2530. f) T. C. Boorman, I. Larrosa, Chem. Soc. Rev. 2011, 40, 1910

[2] a) J. H. Teles, S. Brode, M. Chabanas, Angew. Chem., Int. Ed. 1998, 37, 1415; b) A. S. K. Hashmi, L. Schwarz, J. H. Choi, T. M. Frost, Angew. Chem., Int. Ed. 2000, 39 , 2285 ; c) C. Nevado, D. J. Cardenas, A. M. Echavarren, Chem. Eur. J. 2003, 9, 2627; d) J. J. Kennedy-Smith, S. T. Staben, F. D. Toste, J. Am. Chem. Soc. 2004, 126 , 4526; Reviews: e) A. Fürstner, P. W. Davies, Angew. Chem., Int. Ed. 2007, 46, 3410; f) E. Jimenez-Nunez, A. M. Echavarren, Chem. Comm. 2007, 333; g) S. F. Kirsch, Synthesis 2008, 3183.

[3] E. Jimenez-Nunez, A. M. Echavarren, Chem. Rev 2008, 108,3326

[4] Selected examples: a) H. A. Wegner, S. Ahles, M. Neuburger, Chem. Eur. J. 2008, 14, 11310; b) A. S. K. Hashmi, T. D. Ramamurthi, F. Rominger, J. Organomet. Chem. 2009, 694, 592; c) G. Z. Zhang, Y. Peng, L. Cui, L. M. Zhang, Angew. Chem., Int. Ed. 2009, 48, 3112; d) G. Z. Zhang, L. Cui, Y. Z. Wang, L. M. Zhang, J. Am. Chem. Soc. 2010, 132, 1474; e) G. Z. Zhang, Y. D. Luo, Y. Z. Wang, L. M. Zhang, Angew. Chem., Int. Ed. 2011, 50,4450 ; f) A. D. Melhado, W. E. Brenzovich, A. D Lackner, F. D. Toste, J. Am. Chem. Soc. 2010, 132, 8885 ; g) N. P. Mankad, F. D. Toste, J. Am. Chem. Soc. 2010, 132, 12859; h) W. E. Brenzovich, D. Benitez, A. D. Lackner, H. P. Shunatona, E. Tkatchouk, W. A. Goddard, F. D. Toste, Angew. Chem., Int. Ed. 2010, 49, 5519; i) E. Tkatchouk, N. P. Mankad, D. Benitez, W. A. Goddard, F. D. Toste, J. Am. Chem. Soc. 2011, 133 , 14293; j) T. de Haro, C. Nevado, Angew. Chem., Int. Ed. 2011, 50, 906; k) L. T. Ball, G. C. Lloyd-Jones, C. A Russell, Chem. Eur. J. 2012, 18, 2931; Reviews: 1) P. Garcia, M. Malacria, C. Aubert, V. Gandon, L. Fensterbank, Chemcatchem 2010, 2, 493; m) M. N. Hopkinson, A. D. Gee, V. Gouverneur, Chem. Eur. J. 2011, 17, 8248; n) H. A. Wegner, M. Auzias, Angew. Chem., Int. Ed. 2011, 50, 8236.

[5] F. Diederich, P. J. Stang, R. R. Tykwinski, in Acetylene Chemistry: Chemistry, Biology and Material Science; Wiley-VCH, Weinheim, 2005.

[6] Y. Fuchita, Y. Utsunomiya, M. Yasutake, J. Chem. Soc., Dalton Trans. 2001, 2330.

[7] M. T. Reetz, K. Sommer, Eur. J. Org. Chem. 2003, 3485

[8] P. C. Hayes, S. H. Algie, Process Principles in Minerals and Materials Production, Hayes publishing, Brisbane, 1993

[9] H. Plenio, Angew. Chem., Int. Ed. 2008, 47, 6954.

[10] a) C. González-Arellano, A. Abad, A. Corma, H. García, M. Iglesias, F. Sánchez, Angew. Chem., Int. Ed. 2007, 46, 1536 ; b) A. Corma, C. Gonzalez-Arellano, M. Iglesias, S. Perez-Ferreras, F. Sanchez, Synlett 2007, 1771 ; c) C. Gonzalez-Arellano, A. Corma, M. Iglesias, F. Sanchez, Eur. J. Inorg. Chem. 2008, 1107.

[11] P. H. Li, L. Wang, M. Wang, F. You, Eur. J. Org. Chem. 2008, 5946.

[12] a) L. A. Jones, S. Sanz, M. Laguna, Catal. Today 2007, 122, 403; b) B. Panda, T. K. Sarkar, Tetrahedron Lett. 2010, 51, 301; c) B. Panda, T. K. Sarkar, Chem. Comm. 2010, 46, 3131; d) M. Pena-Lopez, M. Ayan-Varela, L. A. Sarandeses, J. P. Sestelo, Chem. Eur. J. 2010, 16, 990 ; e) S. Pankajakshan, T. P. Loh, Chem. Asian J. 2011, 6, 2291; f) W. Y. Man, S. Bock, N. N. Zaitseva, M. I. Bruce, P. J. Low, J. Organomet. Chem. 2011, 696, 2172; g) B. Panda, T. K. Sarkar, Synthesis 2013, 45, 817.

[13] T. Lauterbach, M. Livendahl, A. Rosellon, P. Espinet, A. M. Echavarren, Org. Lett. 2010, 12, 3006.

[14] a) S. K. Beaumont, G. Kyriakou, R. M. Lambert, J. Am. Chem. Soc. 2010, 132, 12246; b) V. K. Kanuru, G. Kyriakou, S. K. Beaumont, A. C. Papageorgiou, D. J. Watson, R. M. Lambert, J. Am. Chem. Soc. 2010, 132, 8081 ; c) G. Kyriakou, S. K. Beaumont, S. M. Humphrey, C. Antonetti, R. M. Lambert, Chemcatchem 2010, 2 ,
1444; See also: d) M. Livendahl, P. Espinet, A. M. Echavarren, Platinum Metals Rev. 2011, 55, 212.

[15] a) A. Corma, R. Juarez, M. Boronat, F. Sanchez, M. Iglesias, H. Garcia, Chem. Comm. 2011, 47, 1446 ; b) M. Boronat, D. Combita, P. Concepcion, A. Corma, H. Garcia, R. Juarez, S. Laursen, J. D. Lopez-Castro, J. Phys. Chem. C 2012, 116, 24855.

[16] P. S. D. Robinson, G. N. Khairallah, G. Silva, H. Lioe, R. A. J. O'Hair, Angew. Chem., Int. Ed. 2012, 51, 3812.

[17] a) A. S. Dudnik, V. Gevorgyan, V. Angew. Chem., Int. Ed. 2010, 49, 2096; b) S. Messaoudi, J. D. Brion, M. Alami, Eur. J. Org. Chem. 2010, 6495; c) J. P. Brand, J. Waser, Chem. Soc. Rev. 2012, 41, 4165.

[18] Selected examples: a) K. Kobayashi, M. Arisawa, M. Yamaguchi, J. Am. Chem. Soc. 2002, 124, 8528; b) I. V. Seregin, V. Ryabova, V. Gevorgyan, J. Am. Chem. Soc. 2007, 129, 7742; c) Y. H. Gu, X. M. Wang, Tetrahedron Lett. 2009, 50, 763; d) M. Tobisu, Y. Ano, N. Chatani, Org. Lett. 2009, 11, 3250; e) N. Matsuyama, K. Hirano, T. Satoh, M. Miura, Org. Lett. 2009, 11, 4156; f) F. Besselievre, S. Piguel, Angew. Chem., Int. Ed. 2009, 48, 9553; g) S. H. Kim, S. Chang, Org. Lett. 2010, 12, 1868; h) L. Yang, L. A. Zhao, C. J. Li, Chem. Commun. 2010, 46, 4184; i) Y. Wei, H. Q. Zhao, J. Kan, W. P. Su, M. C. Hong, J. Am. Chem. Soc. 2010, 132, 2522; j) Y. Ano, M. Tobisu, N. Chatani, Org. Lett. 2012, 14, 354; k) J. L. G. Ruano, J. Aleman, L. Marzo, C. Alvarado, M. Tortosa, S. Diaz-Tendero, A. Fraile, Angew. Chem., Int. Ed. 2012, 51, 2712; 1) X. Jie, Y. Shang, P. Hu, W. Su, Angew. Chem., Int. Ed. 2013, 52, 3630.

[19] a) V. V. Zhdankin, P. J. Stang, Tetrahedron 1998, 54, 10927; b) V. V. Zhdankin, P. J. Stang, Chem. Rev. 2008, $108,5299$.

[20] a) J. P. Brand, J. Charpentier, J. Waser, Angew. Chem., Int. Ed. 2009, 48, 9346; TIPS-EBX (15) is commercially available or can be easily synthesized from 2-iodo benzoic acid: b) V. V. Zhdankin, C. J. Kuehl, A. P. Krasutsky, J. T. Bolz, A. J. Simonsen, J. Org. Chem. 1996, 61, 6547; c) J. P. Brand, J. Waser, Synthesis 2012, 44, 1155; d) M. J. Bouma, B. Olofsson, Chem. Eur. J. 2012, 18, 14242 .

[21] J. P. Brand, C. Chevalley, R. Scopelliti, J. Waser, Chem. Eur. J. 2012, 18,5655 .

[22] H. Mayr, A. R. Ofial, J. Phys. Org. Chem. 2008, 21, 584.

[23] Y. Li, J. P. Brand, J. Waser, Angew. Chem., Int. Ed. 2013, 52, 6743 .

[24] J. P. Brand, J. Waser, Org. Lett. 2012, 14, 744.

[25] A. Mishra, C. Q. Ma, P. Bauerle, Chem. Rev. 2009, 109, 1141 .

[26] a) R. Koller, K. Stanek, D. Stolz, R. Aardoom, K. Niedermann, A. Togni, Angew. Chem., Int. Ed. 2009, 48, 4332; b) A. E. Allen, D. W. C. MacMillan, J. Am. Chem. Soc. 2010, 132, 4986.

[27] N. Krause, C. Winter, Chem. Rev. 2011, 111, 1994.

[28] J. P. Brand, C. Chevalley, J. Waser, Beilstein J. Org. Chem. 2011, 7, 565.

[29] T. de Haro, C. Nevado, J. Am. Chem. Soc. 2010, 132, 1512 .

[30] M. N. Hopkinson, J. E. Ross, G. T. Giuffredi, A. D. Gee, V. Gouverneur, Org. Lett. 2010, 12, 4904.

[31] D. Y. Qian, J. L. Zhang, Beilstein J. Org. Chem. 2011, 7, 808.

[32] a) A. Domenech, A. Leyva-Perez, S. I. Al-Resayes, A. Corma, Electrochem. Commun. 2012, 19, 145; b) A. Leyva-Perez, A. Domenech, S. I. Al-Resayes, A. Corma, Acs Catalysis 2012, 2, 121

[33] M. Zhu, M. Ning, W. J. Fu, C. Xu, G. L. Zou, Bull. Korean Chem. Soc. 2012, 33, 1325 .

Received: ((will be filled in by the editorial staff))

Accepted: ((will be filled in by the editorial staff)) Published online: ((will be filled in by the editorial staff)) 
Graphical abstract:

\begin{tabular}{|c|c|}
\hline Nucleophilic $\quad \mathbf{R}^{1}-\mathbf{X}+\equiv-R$ & \\
\hline Electrophilic $\mathrm{R}^{1}-\mathrm{H}+\mathrm{X}=\mathrm{R}$ & $\begin{array}{c}A u \\
\text { Catalyzed }\end{array}$ \\
\hline Oxidative $\mathrm{R}^{1}-\mathrm{H}+\equiv \mathrm{R}+[\mathrm{O}]$ & \\
\hline
\end{tabular}

